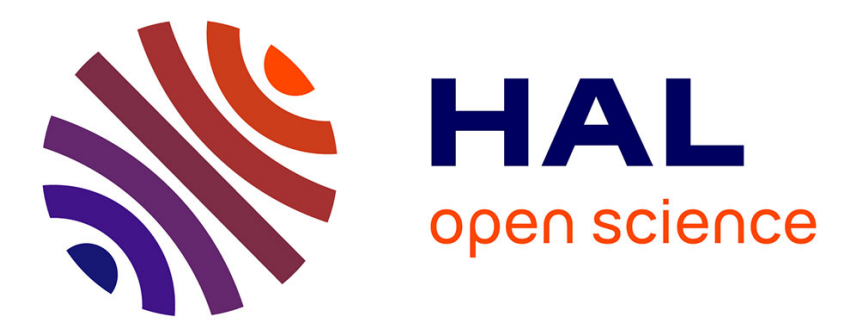

\title{
Dynamics, Fragility, and Glass Transition of Low-Molecular-Weight Linear Homopolymers
}

Laura Andreozzi, Ciro Autiero, Massimo Faetti, Marco Giordano, Fabio Zulli

\section{To cite this version:}

Laura Andreozzi, Ciro Autiero, Massimo Faetti, Marco Giordano, Fabio Zulli. Dynamics, Fragility, and Glass Transition of Low-Molecular-Weight Linear Homopolymers. Philosophical Magazine, 2008, 88 (33-35), pp.4151-4159. 10.1080/14786430802468231 . hal-00513975

\section{HAL Id: hal-00513975 \\ https://hal.science/hal-00513975}

Submitted on 1 Sep 2010

HAL is a multi-disciplinary open access archive for the deposit and dissemination of scientific research documents, whether they are published or not. The documents may come from teaching and research institutions in France or abroad, or from public or private research centers.
L'archive ouverte pluridisciplinaire HAL, est destinée au dépôt et à la diffusion de documents scientifiques de niveau recherche, publiés ou non, émanant des établissements d'enseignement et de recherche français ou étrangers, des laboratoires publics ou privés. 


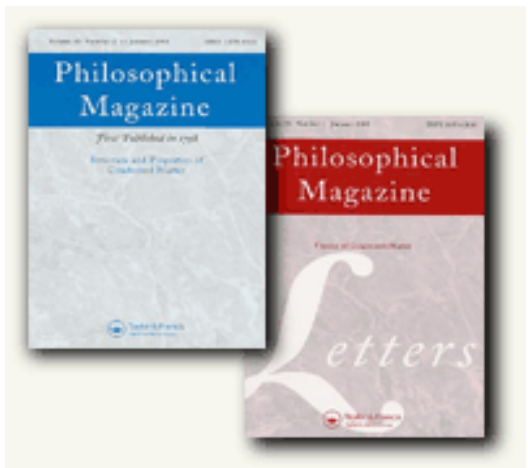

Dynamics, Fragility, and Glass Transition of Low-Molecular-Weight Linear Homopolymers

\begin{tabular}{|r|l|}
\hline Journal: & Philosophical Magazine \& Philosophical Magazine Letters \\
\hline Manuscript ID: & TPHM-08-May-0157.R1 \\
\hline $\begin{array}{r}\text { Date Submitted by the } \\
\text { Author: }\end{array}$ & O2-Sep-2008 \\
\hline Complete List of Authors: & $\begin{array}{l}\text { Andreozzi, Laura; University of Pisa, Physics } \\
\text { Autiero, Ciro; University of Pisa, Physics } \\
\text { Faetti, Massimo; University of Pisa, Physics } \\
\text { Giordano, Marco; University of Pisa, Physics } \\
\text { Zulli, Fabio; University of Pisa, Physics }\end{array}$ \\
\hline Keywords: & glass transition, polymers \\
\hline Keywords (user supplied): & fragility, molecular weight, free volume \\
\hline & \\
\hline $\begin{array}{l}\text { Note: The following files were submitted by the author for peer review, but cannot be converted } \\
\text { to PDF. You must view these files (e.g. movies) online. }\end{array}$ \\
\hline ANDALO.tex
\end{tabular}

\section{今 scholaroNE" \\ Manuscript Central}


Philosophical Magazine

Vol. 00, No. 00, 00 April 2008, 1-8

\title{
RESEARCH ARTICLE
}

\author{
Dynamics, Fragility, and Glass Transition of \\ Low-Molecular-Weight Linear Homopolymers
}

\author{
Laura Andreozzi, ${ }^{*}$ Ciro Autiero, Massimo Faetti, Marco Giordano, Fabio Zulli \\ Department of Physics "E. Fermi", University of Pisa, and polyLab CNR-INFM, \\ largo B. Pontecorvo 3, 56127 Pisa (Italy) \\ ( $v 4.0$ released January 2008)
}

\begin{abstract}
Analysis of the dynamics of low-molar-mass linear homopolymers is of importance to understand the mechanisms driving structural relaxation properties from molecular liquids to polymers. Theoretical models assume, in general, the same mobility of the different moieties of the macromolecule, but this hypothesis fails for low-molar-mass polymers. Therefore, isofrictional comparisons are necessary to interpret experimental data with theoretical models. In this work, the molar-mass dependence of the glass transition temperatures $T_{g}$, fragility indexes, and dynamic moduli of unentangled poly(ethyl acrylate) (PEA) melts are studied and discussed. All the results are interpreted in a coherent framework developed from the freevolume concept. The predictive power of this framework is also tested to fit theoretical and experimental master curves introducing an "inverse" isofrictional correction.
\end{abstract}

Keywords: glass transition; polymers; fragility; molecular weight; free volume.

\section{Introduction}

The study of the dynamics of low-molar-mass linear homopolymers is particularly relevant to understand the mechanisms which drive structural relaxation properties from molecular liquids to polymers. On one hand, the dynamics of polymers and molecular liquids are virtually indistinguishable when approaching the glass transition, which is unaccompanied by marked changes in structure or molecular configuration [1]. On the other hand, the chain connectivity introduces dynamic complexities that make polymer behaviour distinct from that of molecular liquids (e.g. entanglement [2-4]). Therefore, the study and comparison of the mass dependence features of structural relaxation or thermal properties, such as fragility or glass transition temperature $T_{g}$, become essential for the knowledge of those characteristics discriminating the dynamics of molecular liquids, oligomers, and polymers.

In this work, the molar mass dependence of the glass transition temperature $T_{g}$, fragility indexes, and of the dynamic modulus master curve of nearly monodisperse unentangled poly(ethyl acrylate) (PEA) melts are studied and discussed. All the results are interpreted in a coherent framework based on the free-volume concept [5]. This approach also allows one to predict the thermal response, and in particular the friction coefficient, at a given mass value, after having characterised homologue samples in a proper mass range. This capability is of particular importance because assuming the same mobility for different moieties of the macromolecular chain could lead to wrong results when studying the dynamics of low-molar-mass melts [6].

\footnotetext{
*Corresponding author. Email: laura.andreozzi@df.unipi.it. 
In free volume theories [2, and references therein], the difference in chain mobility is justified by an excess of free volume for chain tails. This effect, negligible in high-molar-mass polymers, affects polymer dynamics [6] and also gives rise to the decrease in $T_{g}$ for oligomers described by Fox-Flory law [7]. Free volume theories, even if unable to describe the glass transition phenomenology, have been found to satisfactorily interpret the experimental results of polymer dynamics at room pressure [8]: according to the Williams-Landel-Ferry (WLF) and Vogel-Fulcher (VF) temperature dependence of the structural relaxation times in polymers [5, and references therein], and isofrictional correction for polymer viscosity [9].

In a previous paper [5], we showed how, in a revised free-volume framework, it is possible to interpret the mass dependence of WLF/VF parameters, and how to extend the Fox-Flory law to fit better $T_{g}$ values for the oligomers. In this paper, we show how this model can also be applied to the fragility indexes and extended to other melts such as poly(methyl methacrylate) (PMMA) polymers.

Moreover, it is shown that the iso-free-volume correction is necessary to extract dynamic information from the master curves of the complex shear modulus $G^{*}$ of the unentangled PEA samples studied in this work, and to compare experimental results with the predictions of the Rouse theory for a monodisperse chain [3]. For a monodisperse melt of density $\rho$ and molar mass $M$ it is found [3]:

$$
\begin{gathered}
G_{\text {mono }}^{*}(\omega, M)=\frac{\rho R T}{M} \sum_{p>0}\left(\frac{\omega^{2} \tau_{R}^{2}}{4 p^{4}+\omega^{2} \tau_{R}^{2}}+\mathrm{i} \frac{2 p^{2} \omega \tau_{R}}{4 p^{4}+\omega^{2} \tau_{R}^{2}}\right) \\
\tau_{R}=\frac{\zeta_{0} b^{2} N_{A}}{3 \pi^{2} R T} N^{2}=K_{R} \frac{M^{2}}{M_{0}^{2}} .
\end{gathered}
$$

The Rouse time $\tau_{R}$ depends on the gas constant $R$, the Avogadro number $N_{A}$, the temperature $T$, the number $N$ and rest length $b$ of springs in beads-and-springs model employed to develop Rouse theory [3] and can be written as the product of the square of the ratio of the molar mass of the chain $M$ to the molar mass of a repeating unit $M_{0}$ (where $M / M_{0}=N$ ) and a mass-independent factor $K_{R}$. Besides $T$, the only mass-dependent quantity in the expression of $\tau_{R}$ is the monomeric friction coefficient $\zeta_{0}$, whose temperature dependence is expected to be the same as viscosity $[2,5]$.

The total complex shear modulus $G^{*}$ is given by the contribution of the overall monodisperse components $G_{\text {mono }}^{*}(\omega, M)$ of the molar mass distribution $w(M)=$ $\mathrm{d} W(M) / \operatorname{dlog} M$, where $W(M)$ is the weight fraction of chains with molar mass lower than $M$ :

$$
G(\omega)=\int w(M) G_{\mathrm{mono}}^{*}(\omega, M) \operatorname{dlog} M
$$

\section{Materials and Experiments}

Five narrowly-distributed PEA samples $\left(M_{0}=100.12 \mathrm{~g} \mathrm{~mol}^{-1}\right)$ of different molar masses (labelled as PEA15R, PEA16R, PEA17R, PEA18R, PEA01R) are studied in this work. These samples belong to a series of PEA linear homopolymers synthesised following an atom transfer radical polymerization [5] using ethyl 2-bromo propionate as initiator, which becomes indistinguishable from ethyl acrylate repeat unit once inserted in the macromolecule. The averages $M_{n}$ and $M_{w}$, and polydispersity $M_{w} / M_{n}$ were determined by size exclusion chromatography using monodisperse polystyrene for the calibration [5] (Table 1). 


\section{September 1,2008 \\ 18:55 Philosophical Magazine ANDALOnero \\ Philosophical Magazine \& Philosophical Magazine Letters}

Philosophical Magazine

1

2

3

4

5

6

7

8

9

10

11

12

13

14

15

16

17

18

19

20

21

22

23

24

25

26

27

28

29

30

31

32

33

34

35

36

37

38

39

40

41

42

43

44

45

46

47

48

49

50

51

52

53

54

55

56

57

58

59

60

Table 1. Mass averages and glass transition temperatures of PEA samples discussed in this work (top lines). Data for other PEA melts of the same series discussed in [5] are also given.

\begin{tabular}{lrrrr}
\hline Sample & $M_{n}\left(\mathrm{~g} \mathrm{~mol}^{-1}\right)$ & $M_{w}\left(\mathrm{~g} \mathrm{~mol}^{-1}\right)$ & $M_{w} / M_{n}$ & $T_{g}(\mathrm{~K})$ \\
\hline PEA15R & 980 & 1200 & 1.22 & 231 \\
PEA16R & 2750 & 3000 & 1.09 & 243 \\
PEA17R & 7300 & 7800 & 1.07 & 247 \\
PEA01R & 7500 & 8250 & 1.10 & 248 \\
PEA18R & 8800 & 9600 & 1.09 & 250 \\
\hline PEA02R & 11650 & 13100 & 1.13 & 252 \\
PEA04R & 18650 & 20200 & 1.08 & 250 \\
PEA05R & 24900 & 26600 & 1.07 & 253 \\
PEA06R & 58200 & 66500 & 1.15 & 253 \\
PEA20R & 120000 & 150000 & 1.25 & 253 \\
\hline
\end{tabular}

Differential calorimetry measurements were carried out by means of a DSC7 Perkin-Elmer calorimeter in order to obtain the glass transition temperatures $T_{g}$ (Table 1). Thermograms were recorded on heating at $10 \mathrm{~K} \mathrm{~min}^{-1}$ after a quench at $40 \mathrm{~K} \mathrm{~min}^{-1}$. Mass distribution and calorimetric data of PEA melts of the same series, with higher molar masses, are also given in Table 1 . All the $T_{g}$ data, obtained according to the onset definition, were found to be $1 \mathrm{~K}$ less than the values obtained by enthalpic definition [10], which is known to be a heating-rate-independent procedure.

A Haake RheoStress RS150H stress-controlled rheometer was employed for the linear viscoelastic characterisation of PEA samples. The cone-plate geometry (35 mm diameter, cone angle $4^{\circ}$ ) was utilized for the PEA15R sample, while a parallel plate system (diameter $20 \mathrm{~mm}$ ) has been chosen for all the other samples. Viscosity and thermal parameters of these samples are widely discussed in [5]. Master curves were obtained by mathematical shifting of experimental isotherm frequency sweeps of the complex modulus at the reference temperature $T_{r}=270 \mathrm{~K}$.

To compute master curves of the complex modulus, a numerical algorithm was written, which minimises the reduced $\chi_{r}^{2}$ function

$$
\chi_{r}^{2}=\sum_{k=1}^{n}\left[\left(\frac{\log \left(G_{\exp }^{\prime}\left(\omega_{k}\right) / G_{\mathrm{th}}^{\prime}\left(\omega_{k}\right)\right)}{0.1 \sqrt{n-2}}\right)^{2}+\left(\frac{\log \left(G_{\mathrm{exp}}^{\prime \prime}\left(\omega_{k}\right) / G_{\mathrm{th}}^{\prime \prime}\left(\omega_{k}\right)\right)}{0.1 \sqrt{n-2}}\right)^{2}\right]
$$

obtained fitting experimental master curves $G_{\text {exp }}^{*}(\omega)$ over $n$ points. A global maximum relative error of $10 \%$ can be reasonably considered in the minimisation. The only free parameter is the mass-independent factor $K_{R}$ of the Rouse time (Eq. 2), whose only unknown quantity is the monomeric friction coefficient $\zeta_{0}[5]$.

\section{Results and Discussion}

In a previous study carried out on this PEA series [5], we have analysed the temperature dependence of the zero-shear viscosity. The equivalence between $\mathrm{VF}$ and WLF was also shown. Moreover, starting from the definitions of the WLF parameters in the framework of the free volume model, laws were introduced expressing their molar mass dependencies:

$$
\begin{aligned}
& c_{1}(M)=\frac{B}{f_{r}(M) \ln 10}=\frac{B}{f_{r_{\infty}} \ln 10}\left(1+\frac{M_{f_{r}}}{M}\right)^{-1} \\
& c_{2}(M)=\frac{f_{r}(M)}{\alpha(M)}=\frac{f_{r_{\infty}}}{\alpha_{\infty}} \frac{M+M_{f_{r}}}{M+M_{\alpha}}
\end{aligned}
$$




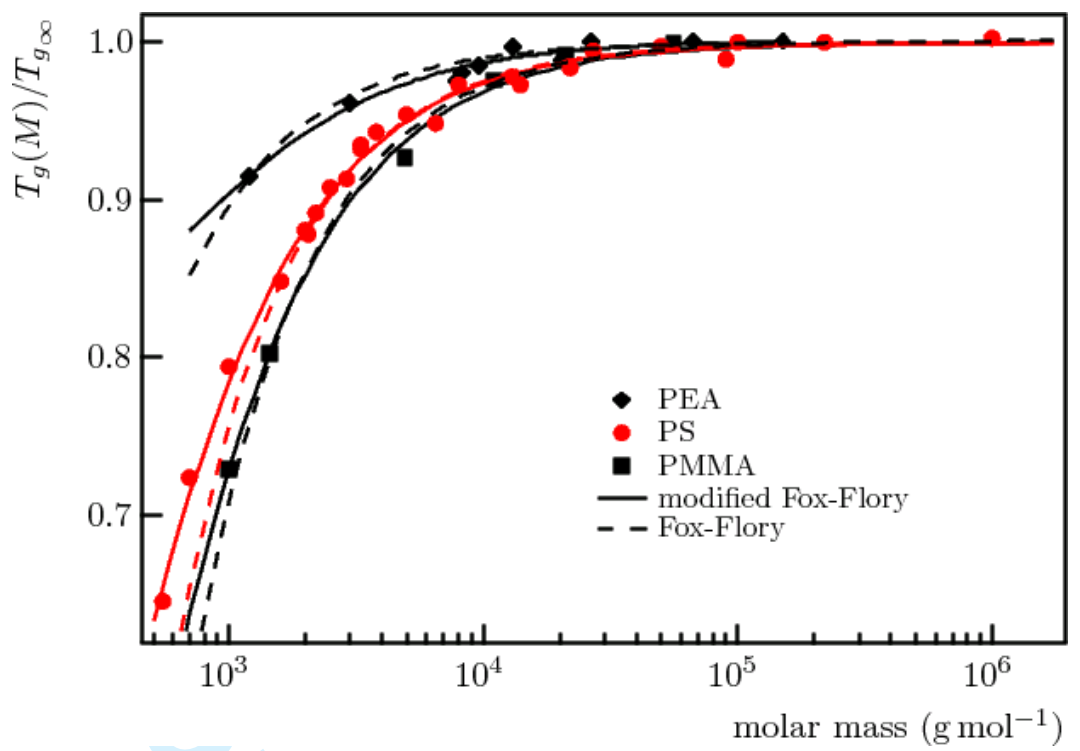

Figure 1. Dependence of the scaled glass transition $T_{g}(M) / T_{g}$ on the molar mass for PEAs and literature series of PS and PMMA [5, 11]. Best fits according to Fox-Flory Eq. 7 (dashed lines) and modified Fox-Flory Eq. 8 (continuous lines) are also shown. Fitting parameters $T_{g_{\infty}}, K^{\prime}$ and $M_{\alpha}$ of Eq. 8 are respectively: $253 \mathrm{~K}, 38000 \mathrm{~K} \mathrm{~mol} \mathrm{~g}^{-1}, 550 \mathrm{~g} \mathrm{~mol}^{-1}$ for PEAs; $369 \mathrm{~K}, 95500 \mathrm{~K} \mathrm{~mol} \mathrm{~g}^{-1}$, $200 \mathrm{~g} \mathrm{~mol}^{-1}$ for PSs; $395 \mathrm{~K}, 127000 \mathrm{~K} \mathrm{~mol} \mathrm{~g}^{-1}, 180 \mathrm{~g} \mathrm{~mol}^{-1}$ for PMMAs.

where $B$ is a constant for homologue polymers, while the free-volume ratio ${ }^{1} f_{r}$ and the thermal expansion coefficient $\alpha$ are mass dependent quantities, which grow and reach constant values at high molar masses (these asymptotic values are labelled with a subscript " $\infty$ "). The mass parameters $M_{f_{r}}$ and $M_{\alpha}$ determine the mass dependence of $f_{r}$ and $\alpha$, respectively. Data of $c_{1}$ were then employed to study the mass dependence of the viscosity in the isofrictional case, obtained applying the literature correction [9]

$$
\zeta_{0}(M)=\zeta_{0} 10^{c_{1}(M)-c_{1}}
$$

With this approach, it is also possible to refine Fox-Flory law [7] for the mass dependence of $T_{g}$ towards its asymptotic value $T_{g_{\infty}}$. In fact, taking account of the tail mobility $\theta$, Fox-Flory law can be written as

$$
T_{g}(M)=T_{g_{\infty}}-\frac{2 \theta \rho N_{A}}{\alpha M}
$$

Quite reasonably, $\theta$ can be considered independent of the molar mass. Usually, also $\alpha$ is considered as a constant, but coherently with the mass dependence of $c_{1} c_{2}=$ $B / \alpha$ observed for PEAs [5], we have suggested that also the mass dependence of $\alpha$ should be made explicit [5]. Thus, a modified Fox-Flory law is easily obtained:

$$
T_{g}(M)=T_{g_{\infty}}-\underbrace{\frac{2 \theta \rho N_{A}}{\alpha_{\infty}}}_{K^{\prime}} \frac{1}{M_{\alpha}+M} .
$$

It is worth noting that the parameter $M_{\alpha}$ can be evaluated indipendently by studying WLF parameters.

\footnotetext{
${ }^{1}$ The volume ratio $f_{r}$ is defined as $v_{f} /\left(v_{f}+v_{o}\right)$ being respectively $v_{f}$ the free volume and $v_{o}$ the occupied
} volume at the temperature $T_{r}$. 


\section{ptember 1,2008
Page $\mathbf{5}$ of $\mathbf{1 3}$

Figure 1 shows the measured values of $T_{g}$ of the PEAs of this work, and literature poly(styrene) (PS) and PMMA series [5, 11, and references therein]. Fitting curves obtained from Eq. 7 and Eq. 8 are also provided for each series. It can be noted that the shape of the original Fox-Flory law generates values lower than the modified Eq. 8 at very low molar masses. This behaviour led Kanig and Uberreiter [12] to introduce the empirical dependence $1 / T_{g}(M)=\left(1 / T_{g_{\infty}}+C / M\right)$ to better describe the $T_{g}(M)$ behaviour in the oligomers studied in their experiment. Nonetheless, effectiveness of the modified Eq. 8 is apparent from inspection of Figure 1. Therefore, its microscopic derivation makes it preferable to the empirical Kanig-Uberreiter expression.

Considerations about free volume at $T_{g}$ lead to state that the free volume ratio at $T_{g}\left(f_{g}\right)$ is a constant, therefore $c_{1}^{g}$ is expected to be independent of the mass (Table 2), while mass dependence of $c_{2}^{g}$ is determined by $\alpha(M)$ :

$$
\begin{aligned}
c_{1}^{g} & =\frac{B}{f_{g} \ln 10} \\
c_{2}^{g}(M) & =\frac{f_{g}}{\alpha_{\infty}}\left(1+\frac{M_{\alpha}}{M}\right)^{-1} .
\end{aligned}
$$

With these expressions, one can evaluate another relevant quantity extrapolated at $T_{g}$, namely the dynamic fragility

$$
m=\left.\frac{\mathrm{d} \log \tau}{\mathrm{d}\left(T / T_{g}\right)}\right|_{T=T_{g}}=\frac{c_{1}^{g}}{c_{2}^{g}} T_{g} .
$$

The quantity $m$ is also referred to as steepness index [13]. Often, $m$ has been correlated to the thermodynamic steepness index, as defined in terms of the variation of the heat capacity at the glass transition. However, no clear connections between thermodynamic and dynamic steepness indexes have been found yet [14-16]. Instead, dynamic fragility can be related to other quantity employed to discriminate between fragile and strong behaviours of glass formers: $D=\ln 10 c_{1}^{g} c_{2}^{g} /\left(T_{g}-c_{2}^{g}\right)$ [1]. In fact, it holds $D\left(m-c_{1}^{g}\right)=\ln 10\left(c_{1}^{g}\right)^{2}$.

Also the fragility of polymers can vary with the molar mass. In the framework sketched in this work, the mass dependence of the parameters $D$ and $m$ can be analysed. It is found:

$$
m(M)=T_{g_{\infty}} \frac{B \alpha_{\infty}}{f_{g}^{2} \ln 10}\left(1+\frac{M_{\alpha}-K^{\prime} / T_{g_{\infty}}}{M}\right) .
$$

It is worth recalling that the parameter $K^{\prime}$ defined in Eq. 8 depends on the tail mobility, and therefore on tail microstructure. The sign of the first derivative of the indexes $m(M)$, in line of principle, could assume any value (positive or negative) depending on the sign of $K^{\prime} / T_{g_{\infty}}-M_{\alpha}$. But for all known cases, the dynamic fragility $m$ has been found to assume direct or null dependence on the molar mass [16] (e.g. direct for poly(styrene) [17], poly(propylene glycol) [18]; null for poly(isoprene) [19], poly(dimethyl siloxane) [20], poly(phenylmethyl siloxane) [20], poly(isobutylene) [21]).

Anyway, it should be remarked that many literature data are affected by experimental drawbacks (e.g. few samples with low molar mass, polymers with high polydispersity, extrapolated key quantities such as $T_{g}$ ). In this sense, the case of PMMA is emblematic. 
Table 2. Dynamic fragility $m, D=\ln 10 c_{1}^{g} c_{2}^{g} /\left(T_{g}-c_{2}^{g}\right)$, and WLF invariant $c_{1}^{g}$ for PEA melts and literature PMMA melts [11]

\begin{tabular}{lccclccc}
\hline Sample & $D$ & $m$ & $c_{1}^{g}$ & Sample & $D$ & $m$ & $c_{1}^{g}$ \\
\hline PEA15R & $5.5 \pm 0.3$ & $78 \pm 7$ & $12.6 \pm 1.1$ & PEA02R & $7.1 \pm 0.3$ & $59 \pm 6$ & $12.0 \pm 0.8$ \\
PEA16R & $6.3 \pm 0.3$ & $65 \pm 7$ & $11.8 \pm 1.0$ & PEA04R & $7.3 \pm 0.3$ & $65 \pm 8$ & $12.7 \pm 0.8$ \\
PEA17R & $6.6 \pm 0.4$ & $62 \pm 7$ & $11.8 \pm 0.8$ & PEA05R & $7.5 \pm 0.3$ & $60 \pm 7$ & $12.0 \pm 0.8$ \\
PEA01R & $6.8 \pm 0.4$ & $62 \pm 9$ & $11.8 \pm 1.2$ & PEA06R & $7.3 \pm 0.3$ & $60 \pm 9$ & $12.1 \pm 1.2$ \\
PEA18R & $6.9 \pm 0.3$ & $63 \pm 7$ & $12.2 \pm 0.8$ & PEA20R & $7.4 \pm 0.3$ & $61 \pm 7$ & $12.2 \pm 0.8$ \\
\hline PMMA1450 & - & 91 & - & PMMA21000 & - & 121 & - \\
PMMA4900 & - & 118 & - & PMMA56000 & - & 121 & - \\
PMMA11000 & - & 115 & - & & & & \\
\hline
\end{tabular}

In fact, even if most authors agree that for PMMA $m(M)$ increases with the mass, very different (often contradicting) values of $m(M)$ are found in the literature $[1,11,17,22$, and references therein]. Source of further ambiguity comes from the comparison of $m$ indexes calculated for different relaxation times, because of their possible different temperature dependence $[8,23]$.

In Table 2, the mass dependence of $D$ and $m$ can be observed for PEA samples and literature PMMA series [11]. It is interesting to observe how these two series fulfil Eq. 11: PEAs show a slightly decreasing $m(M)$, while for PMMAs an increasing $m(M)$ is observed. However, even if the dynamic fragility of PEA15R is above the mean value obtained from the other samples, the null hypothesis for the PEA series cannot be discarded. In fact, fit of experimental data with a constant function provided $\chi^{2}=5.54$, which is well inside the $95 \%$ confidence interval for nine degrees of freedom. It is also worth noting that only the modified Fox-Flory fit of $T_{g}(M)$ (Eq. 8) is needed to determine the mass dependence of fragility $m(M)$ (or $D(M)$ ). Moreover, it can be easily understood how structural and relaxation parameters of melts of any mass can be predicted due to the consistence of this approach. This turns out to be particularly useful when studying polymer dynamics.

We have shown how structural relaxation quantities of low-molar-mass polymers, such as fragility and $T_{g}$, are affected by the chain length. Let us now consider the polymer dynamics and verify that influence of the molar mass of the melts is properly taken into account by theoretical models if differences of the free volume at the chain tails are considered. The complex shear modulus of the unentangled PEA melts studied in this work (PEA15R, PEA16R, PEA17R, PEA01R, and PEA18R) is predicted according to Rouse theory (Eq. 1). Because of the small molar mass of the samples, calculations of complex shear modulus need an isofrictional correction. In fact, in experimental spectra, the contribution of low molar masses differs from the theoretical one because the Rouse model, where isofrictional relaxation times are assumed, does not take proper account of the different chain-tail mobility, while highly mass-dependent $\zeta_{0}$ can be found for chains of low molar masses. Therefore, a correction of the models has to be applied to properly fit the experimental behaviour:

$$
\zeta_{0}(M)=\zeta_{0} 10^{c_{1 \infty}-c_{1}(M)}
$$

Eq. 12 expresses a correction "inverse" with respect to Eq. 6, suggested in [9], which allows experimental data to fit the theoretical behaviour. It is worth recalling that the function $c_{1}(M)$ can be expressed analytically as $M_{f_{r}} c_{1 \infty} /\left(M+M_{f_{r}}\right)$ [5]. The parameters $c_{1_{\infty}}$ and $M_{f_{r}}$ for PEAs can be determined by fitting WLF parameters obtained by the master curve construction at the reference temperature $T_{r}$ [5].

Experimental master curves and superimposed calculated $G^{\prime}$ and $G^{\prime \prime}$ moduli are shown in Figure 2. Relevant fitting parameters are given in Table 3. As one can see, the quality of the fits is very good. 

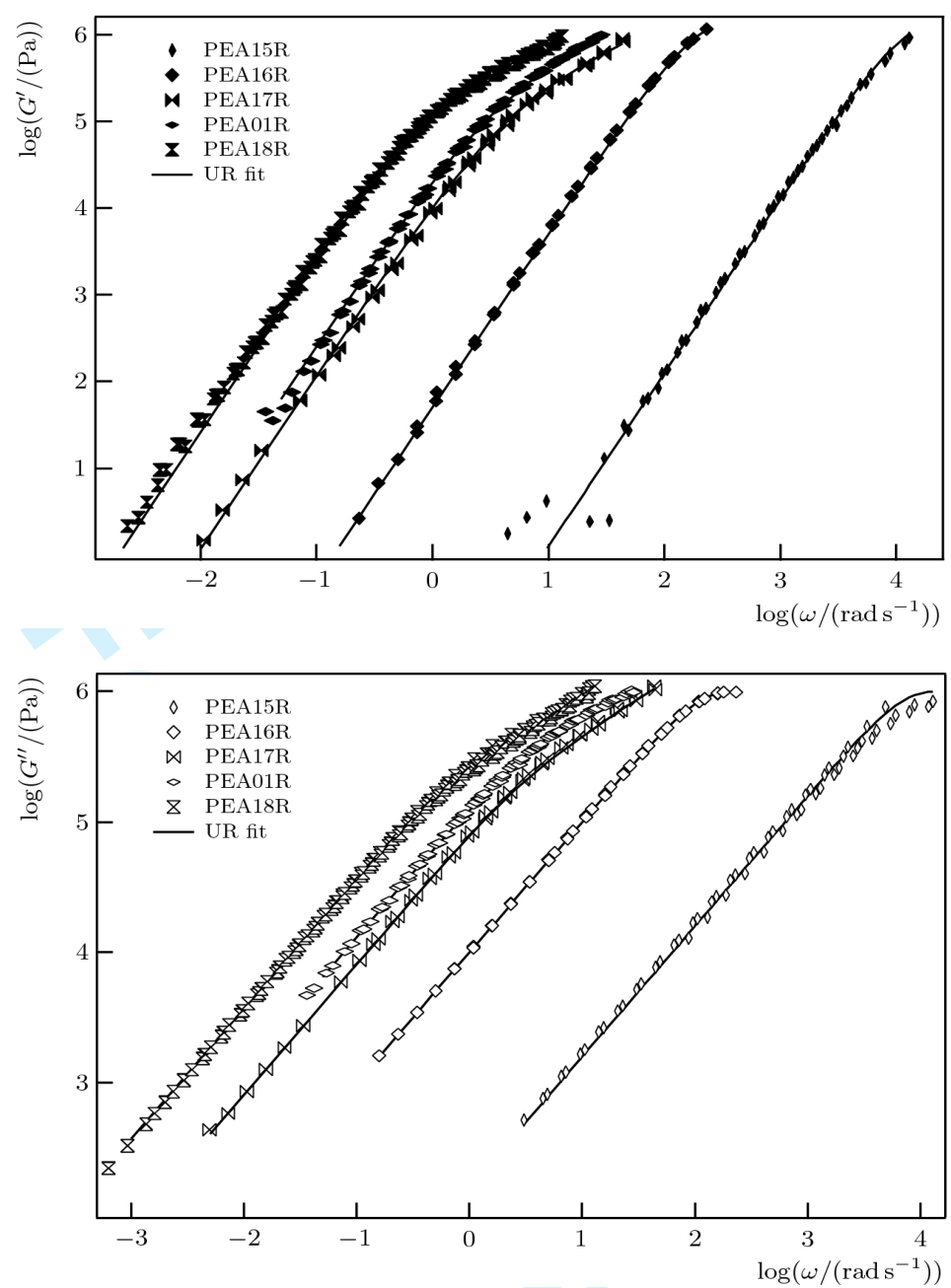

Figure 2. Simulations of master curves of unentangled PEA samples according to the Rouse model (UR). Experimental master curves and superimposed calculated $G^{\prime}$ and $G^{\prime \prime}$ moduli are shown. Relevant fitting parameters are given in Table 3 .

Table 3. Fitting parameters of master curves of unentangled PEA melts. Data given with error are free fitting parameters. The coefficient $K_{R}$ is equal to $\zeta_{0} b^{2} N_{A} /\left(3 \pi^{2} R T\right)$.

\begin{tabular}{lcclcc}
\hline Sample & $K_{R}(\mathrm{~ms})$ & $\chi_{r}^{2}$ & Sample & $K_{R}(\mathrm{~ms})$ & $\chi_{r}^{2}$ \\
\hline PEA15R & $0.76 \pm 0.01$ & 0.30 & PEA01R & $0.80 \pm 0.01$ & 0.52 \\
PEA16R & $0.81 \pm 0.01$ & 0.41 & PEA18R & $0.79 \pm 0.01$ & 0.88 \\
PEA17R & $0.76 \pm 0.01$ & 0.25 & & & \\
\hline
\end{tabular}

It is worth noting that, without the correction of the monomeric friction coefficient suggested in Eq. 12, the calculations would have been still of good quality (Figure 3), but the monomeric friction coefficients would have changed strongly with the mass, due to the different contribution of the tails to the free volume of the melts. For example, iso-free-volume "inverse" correction of the master curves provided the mean value $b^{2} \zeta_{0}=(7.8 \pm 0.2) \times 10^{-23} \mathrm{~N} \mathrm{~s} \mathrm{~m}$, while for PEA17R and PEA18R samples, calculations without the correction provided $b^{2} \zeta_{0}=(6.7 \pm 0.1) \times 10^{-24} \mathrm{~N} \mathrm{~s} \mathrm{~m}$ and $b^{2} \zeta_{0}=(1.98 \pm 0.03) \times 10^{-23} \mathrm{~N} \mathrm{~s} \mathrm{~m}$, respectively. Without performing corrections for the master curves, one would have assumed the overall monomeric friction coefficient, which includes the tail effects, as the friction coefficient of each dynamic unit of the chain, including those inside the polymer backbone, which indeed have a lower mobility. 


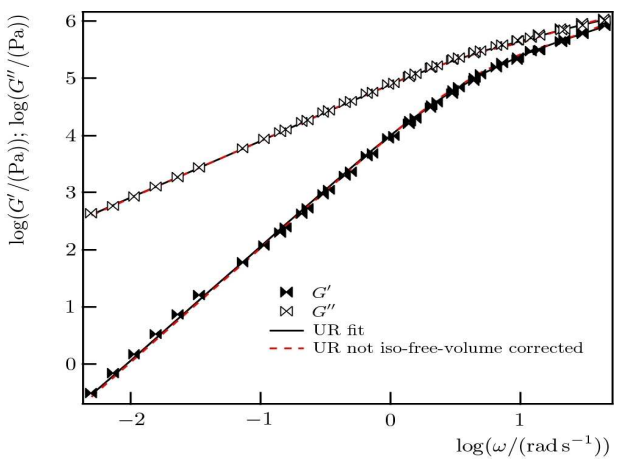

(a)

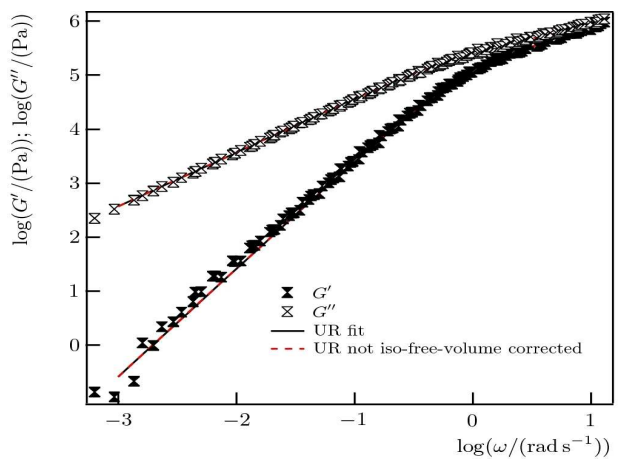

(b)

Figure 3. Calculated $G^{*}$ with and without iso-free-volume correction according to the Rouse model (UR). The cases of PEA17R (a) and PEA18R (b) are shown. Quality of the fits is acceptable in both cases, but $\zeta_{0}$ is not a constant when the Rouse model is not corrected by Eq. 12 .

\section{Conclusions}

In this work, we have shown how the mass dependence of the glass transition temperature and fragility indexes can be interpreted in a coherent framework developed from the free volume. This approach has been also tested on other literature polymer series, namely PS and PMMA. The way in which the chain-tail concentration in low-molar-mass polymers affects chain dynamics has been also discussed. In particular, in order to fit experimental data and theoretical models for polymer dynamics, usually based on a mass-independent monomeric friction coefficient, an "inverse" iso-free-volume correction has been suggested. Such a correction allows one to determine correct microscopic information about chain dynamics also for low-molar-mass macromolecules.

\section{Acknowledgements}

We thank Regione Toscana POR OB3 D4 for partial financial support of the work.

\section{References}

[1] R. Casalini, C.M. Roland, and S. Capaccioli, J. Chem. Phys. 126 (2007), p. 184903.

[2] J.D. Ferry Viscoelastic Properties of Polymers, Wiley, New York, 1980.

[3] M. Doi and S.F. Edwards The Theory of Polymer Dynamics, Oxford University Press, Oxford, 1986.

[4] L. Andreozzi et al., Phil. Mag. 84 (2004), pp. 1555-1565.

[5] L. Andreozzi et al., Macromolecules 39 (2006), pp. 1880-1889.

[6] C.G. Robertson and C.M. Roland, J. Polym. Sci., Part B: Polym. Phys., 42 (2004), pp. $2604-2611$.

[7] T.G. Fox and P.J. Flory, J. Appl. Phys. 21 (1950), p. 581.

[8] K.L. Ngai and D.J. Plazek, Rubber Chem. Technol. 68 (1995), pp. 376-434.

[9] R.H. Colby, L.J. Fetters, and W.W. Graessley, Macromolecules 20 (1987), p. 2226.

[10] M.J. Richardson and N.G. Savill, Polymer 16 (1975), pp. 753-757.

[11] L. Andreozzi et al., Macromolecules 38 (2005), pp. 6056-6067.

[12] G. Kanig and K. Uberreiter, J. Colloid. Sci. 7 (1952), p. 569.

[13] D.J. Plazek and K.L. Ngai, Macromolecules 24 (1991), pp. 1222-1224.

[14] D. Huang and G.B. McKenna, J. Chem. Phys. 114 (2001), p. 5621.

[15] L. Andreozzi et al., J. Phys.-Condens. Matter 15 (2003), pp. S1215-S1226.

[16] A.P. Sokolov, V.N. Novikov, and Y. Ding, J. Phys.-Condens. Matt. 19 (2007), p. 205116.

[17] Y. Ding et al., Macromolecules 37 (2004), pp. 9264-9272.

[18] C. Leon, K.L. Ngai, and C.M. Roland, J. Chem. Phys. 110 (1999), p. 11585.

[19] M. Abdel-Goad et al., Macromolecules 37 (2004), pp. 8135-8144.

[20] S. Pawlus et al., Rubber Chem. Tech. 76 (2003), p. 1106.

[21] K. Kunal et al., J. Polym. Sci., Part B: Polym. Phys., 46 (2008), pp. 1390-1399.

[22] K. Fuchs, C. Friedrich, and J. Weese, Macromolecules 29 (1996), pp. 5893-5910.

[23] C.M. Roland et al., Macromolecules 34 (2001), pp. 6159-6160. 


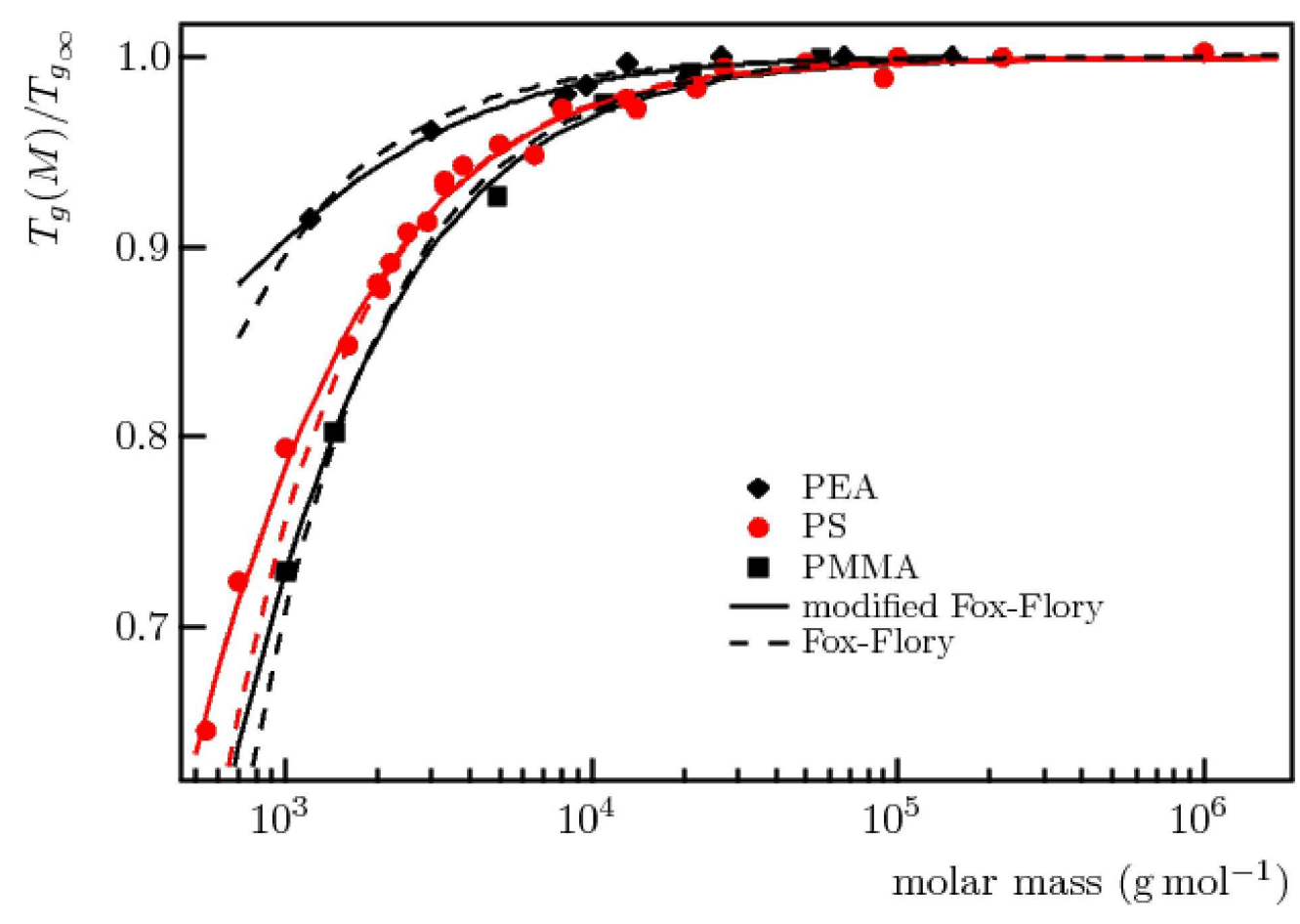

$161 \times 111 \mathrm{~mm}(600 \times 600 \mathrm{DPI})$ 


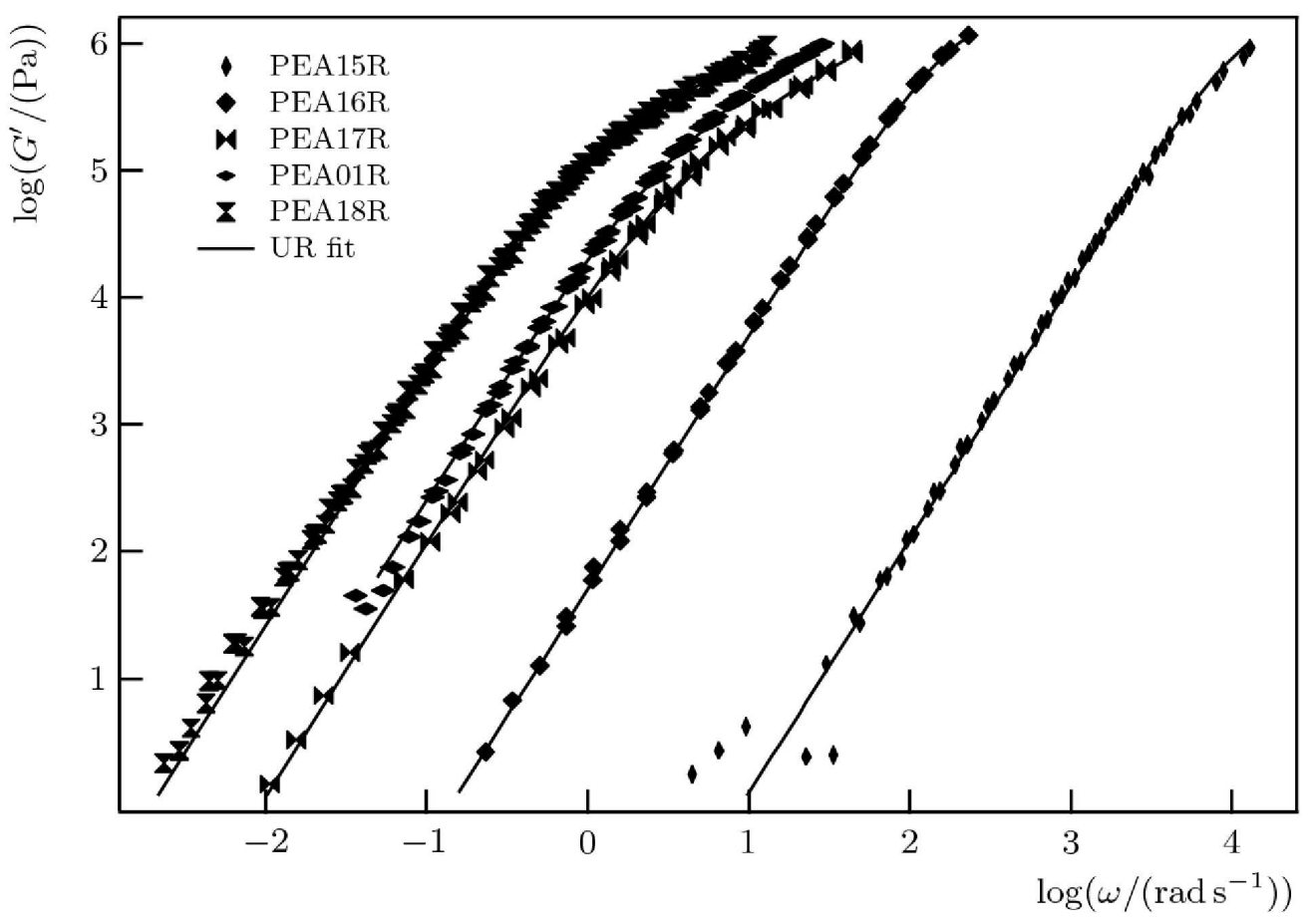

$325 \times 225 \mathrm{~mm}(600 \times 600 \mathrm{DPI})$ 


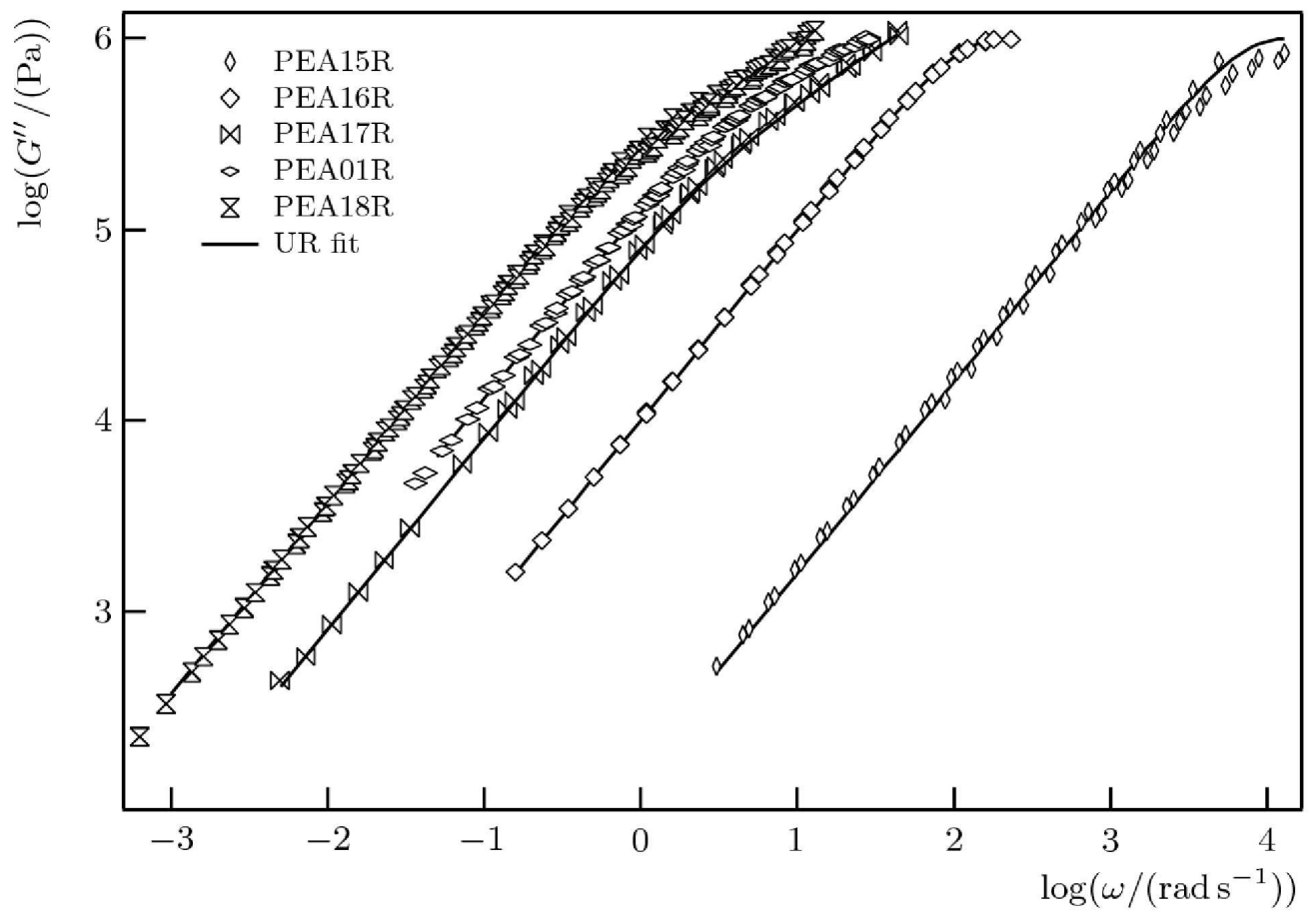

$325 \times 225 \mathrm{~mm}(600 \times 600 \mathrm{DPI})$ 


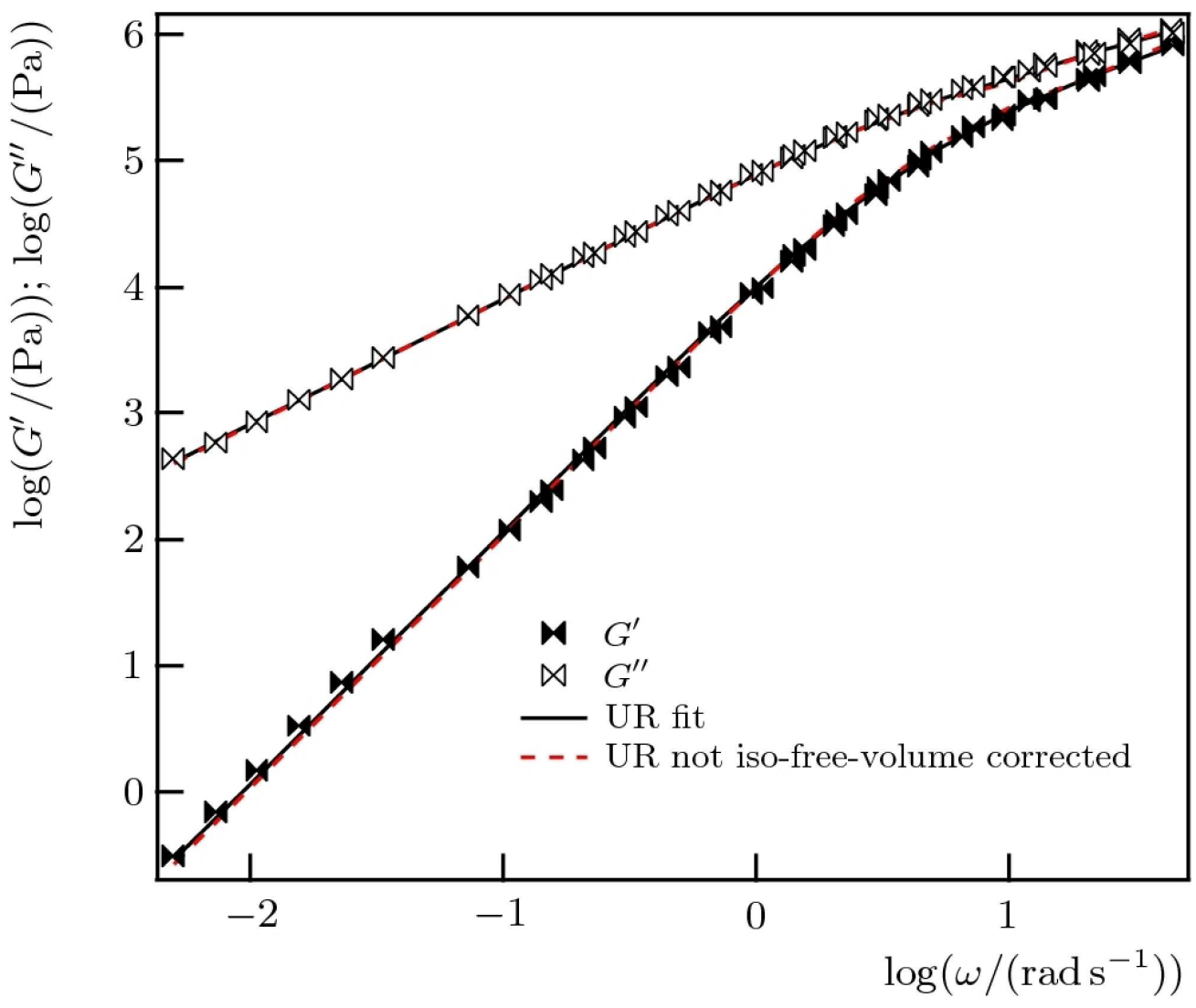

$254 \times 211 \mathrm{~mm}(600 \times 600 \mathrm{DPI})$ 


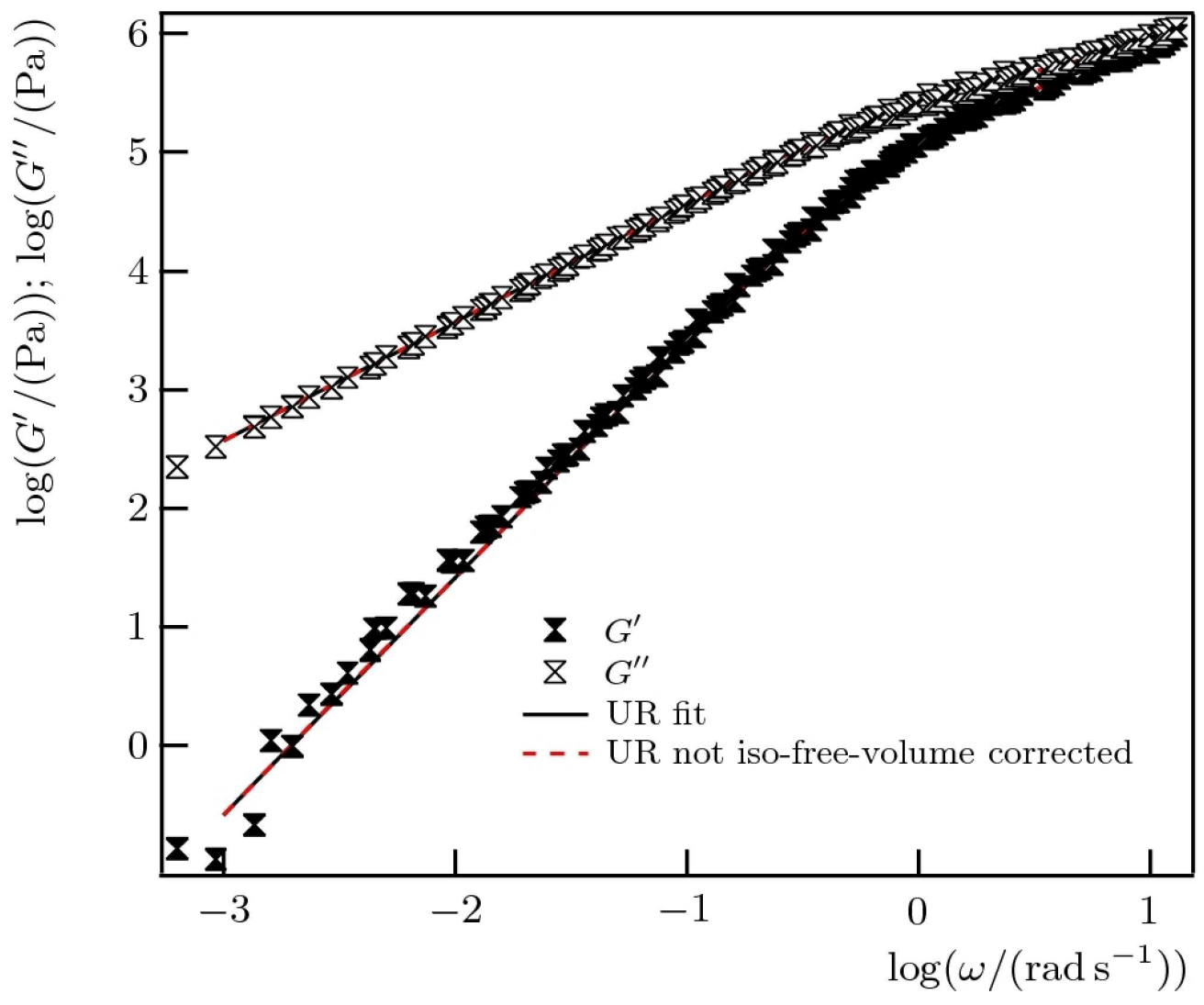

$256 \times 211 \mathrm{~mm}(600 \times 600 \mathrm{DPI})$ 